\title{
Carrier lifetimes in strained InGaAs/(AI)GaAs multiple quantum wells
}

\author{
M. H. Moloney and J. Hegarty \\ Optronics Ireland Research Centre, Department of Pure and Applied Physics, Trinity College, Dublin 2,
Ireland \\ L. Buydens and P. Demeester \\ I.M.E.C., Rijkuniversiteit Gent, Sint-Pieternieuwstraat, 41, B9000 Gent, Belgium \\ R. Grey and J. Woodhead \\ Department of Electrical and Electronic Engineering, University of Sheffield, Mappin Street, Sheffield SI \\ 3JD, United Kingdom
}

(Received 18 January 1993; accepted for publication 2 April 1993)

\begin{abstract}
The effect of strain, strain relief, and barrier design on the carrier lifetime in InGaAs/(A1) GaAs multiple-quantum-well samples is investigated. Carriers lifetimes are measured in samples with varying amount of strain, due to increasing indium concentration in the wells, as well as in samples subject to strain relief, with thick barriers and GaAs barriers. Lifetimes of the order of $0.5 \mathrm{~ns}$ are measured. The lifetime is sensitive to the presence of indium in the wells but remarkably insensitive to the indium concentration, the strain in the samples, and the barrier composition.
\end{abstract}

Many optical devices such as diode lasers and modulators, exploiting both electro-optic ${ }^{1,2}$ and all optical nonlinearities, ${ }^{3}$ have been demonstrated with InGaAs grown on GaAs as the active layer. These devices have very good operating characteristics, despite the considerable lattice mismatch inherent in their growth, and even though short carrier lifetimes have been recorded, 4,5 strained lasers have shown very low threshold currents. ${ }^{6.7}$ The short lifetimes may be a result of the strain, or strain relief through lattice dislocations, but despite the growing importance of these materials very little effort has been made to understand the carrier dynamics in InGaAs quantum wells. We present in this letter a systematic investigation of carrier lifetimes as a function of strain, through varying the indium concentration from $0 \%$ to $15 \%$, and as a function of strain relief and barrier design. The lifetimes are uniformly short, of the order of $0.5 \mathrm{~ns}$, very sensitive to the introduction of indium into the wells, but particularly insensitive to the strain and the design of the active layer. We attribute the short lifetimes to indium-related effects which are present even at small indium concentrations.

Six samples were designed for this investigation, with the seventh being an InGaAs/GaAs asymmetric FabryPerot modulator (AFPM) that has been previously reported on. ${ }^{2,3}$ We shall refer to the samples as $\mathrm{S} 1-\mathrm{S} 7$ as detailed in Table I. S1-S6 were grown by metal organic vapor phase epitaxy (MOVPE) on an undoped GaAs substrate starting with $0.5 \mu \mathrm{m}$ of GaAs followed by $0.5 \mu \mathrm{m}$ of $\mathrm{Al}_{0.35} \mathrm{Ga}_{0.65} \mathrm{As}$ and $0.5 \mu \mathrm{m}$ of $\mathrm{Al}_{0.2} \mathrm{Ga}_{0.8} \mathrm{As}$, the last $1 \mu \mathrm{m}$ of AlGaAs acting as an etch stop. On top of these latticematched layers were grown $\mathbf{P}$ periods of $8 \mathrm{~nm} / 8 \mathrm{~nm}$ $\mathrm{In}_{\mathbf{x}} \mathrm{Ga}_{1-\mathrm{x}} \mathrm{As} / \mathrm{Al}_{0.2} \mathrm{Ga}_{0.8} \mathrm{As}$ well/barrier pairs. Values for $\mathbf{P}$ and $x$ for each of the samples are listed in Table I. S6 had thicker barriers of $25 \mathrm{~nm} \mathrm{Al} \mathrm{A}_{0.2} \mathrm{Ga}_{0.8}$ As. The wafers were capped with $0.5 \mu \mathrm{m}$ of $\mathrm{Al}_{0.2} \mathrm{Ga}_{0.8} \mathrm{As}$. The whole structure was intrinsically doped. The AFPM (S7) was grown by molecular beam epitaxy (MBE) with a 15.5 period $n$-type $\mathrm{GaAs} / \mathrm{AlAs}$ dielectric stack on top of which were grown 29.5 periods of intrinsic $15 \mathrm{~nm} / 15 \mathrm{~nm} \mathrm{In}_{0.1} \mathrm{Ga}_{0.9} \mathrm{As} / \mathrm{GaAs}$ quantum well/barrier pairs followed by a $0.2 \mu \mathrm{m} p$-type GaAs cap.

In order to investigate the effect of increasing indium concentration and the resulting increase in strain on the carrier lifetimes, S1, S2, S3, and S4 were grown with indium concentrations of $0 \%-15 \%$, keeping all other parameters constant. The lattice mismatch in these samples was estimated to be $0 \%, 0.36 \%, 0.71 \%$, and $1.06 \%$, respectively. S4 and S5 were grown to investigate the effects of partial strain relief which results from the thicker structure approaching the critical thickness. ${ }^{8}$ The effects of having thicker barrier structures, as described later, dictated the design of S6.

Normalized absorption spectra for samples S4 and S5 are shown in Fig. 1. These spectra show clear and strong exciton resonances at 927 and $933 \mathrm{~nm}$ for S4 and S5, respectively. Although S4 and S5 differ only in the number of well/barrier periods, the observable shift in the exciton positions, indicating a decrease in band gap, shows that strain relief is significant. ${ }^{9}$ In Fig. 1, for wavelengths below the exciton features, the absorption can be seen to increase rapidly due to residual absorption of the $400 \mu \mathrm{m} \mathrm{GaAs}$ substrate. In S4, S5, and S6 the position of the exciton on the GaAs edge did allow nonlinear measurements to be

TABLE I. Design details and lifetimes of samples.

\begin{tabular}{|c|c|c|c|c|c|c|}
\hline $\begin{array}{l}\text { Sample } \\
\text { code }\end{array}$ & $\begin{array}{l}\text { Indium } \\
\text { conc. } \\
\mathbf{x}\end{array}$ & $\begin{array}{l}\text { No. of } \\
\text { periods } \\
\quad P\end{array}$ & $\begin{array}{l}\text { Well/ } \\
\text { barrier } \\
\text { width } \\
(\mathrm{nm})\end{array}$ & $\begin{array}{c}\text { Barrier } \\
\text { composition }\end{array}$ & $\begin{array}{c}\text { Pump-probe } \\
\text { wavelength } \\
\text { (nm) }\end{array}$ & $\begin{array}{c}\text { Lifctime } \\
\text { (ns) }\end{array}$ \\
\hline S1 & 0.00 & 8 & $8 / 8$ & $\mathrm{Al}_{0.2} \mathrm{Ga}_{0.8} \mathrm{As}$ & 839.5 & 3.98 \\
\hline S2 & 0.05 & 8 & $8 / 8$ & $\mathrm{Al}_{0.2} \mathrm{Ga}_{0.8} \mathrm{As}$ & 857.5 & 0.440 \\
\hline S3 & 0.10 & 8 & $8 / 8$ & $\mathrm{Al}_{0.2} \mathrm{Ga}_{0.8} \mathrm{As}$ & 892.5 & 0.558 \\
\hline S4 & 0.15 & 8 & $8 / 8$ & $\mathrm{Al}_{0.2} \mathrm{Ga}_{0.8} \mathrm{As}$ & 927 & $\begin{array}{l}0.553(\mathrm{a}) \\
0.552(\mathrm{~b})\end{array}$ \\
\hline S5 & 0.15 & 40 & $8 / 8$ & $\mathrm{Al}_{0.2} \mathrm{Ga}_{0.8} \mathrm{As}$ & 933 & 0.564 \\
\hline S6 & 0.15 & 40 & $8 / 25$ & $\mathrm{Al}_{0.2} \mathrm{Ga}_{0.8} \mathrm{As}$ & 933 & 0.570 \\
\hline S7 & 0.10 & 29.5 & $15 / 15$ & GaAs & 950 & 0.440 \\
\hline
\end{tabular}




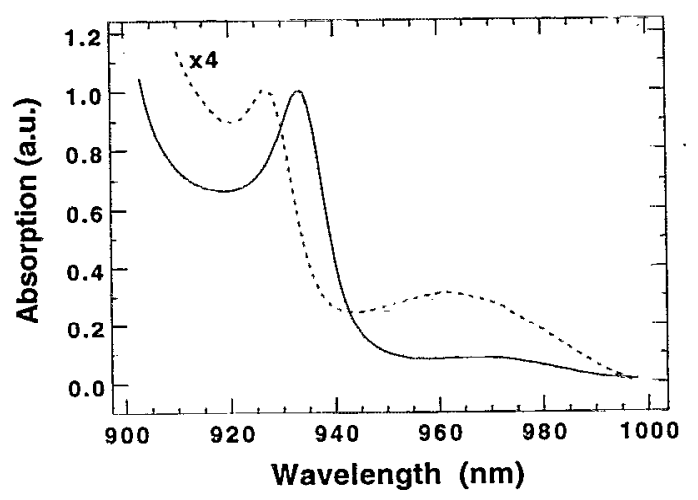

FIG. 1. Absorption spectra from $\mathrm{S} 4$, dashed line, and $\mathrm{S} 5$, full line, show clear strong heavy hole excitons at $927 \mathrm{~nm}(1.3375 \mathrm{eV})$ and $933 \mathrm{~nm}$ $(1.3288 \mathrm{eV})$. As there are only 8 wells in $\mathrm{S} 4$ its spectrum is increased by a factor of 4 so as to clearly show the shift in exciton position. The rise in signals on long wavelength side of exciton features, around $960-970 \mathrm{~nm}$, is due to Fabry-Perot effects that show up in the absorption measurement. Absorption can be seen to rapidly increase below $910 \mathrm{~nm}$ due to residual GaAs substrate absorption.

made as a clear exciton was visible in transmission. Indium concentrations of $0 \%-10 \%$ with $8 \mathrm{~nm}$ wells result in the exciton feature at room temperature being buried in the GaAs residual absorption. To make transmission measurements on these samples the substrate was removed from S1, S2, and S3 using a plasma etching system. To assess the effects of removing all the substrate of the strained layers $S 4$ was cleaved into $S 4 a$ and $S 4 b$, with $S 4 a$ etched and $S 4 b$ left unetched. Measurements made on the AFPM were made in reflection.

Lifetimes were measured using a pump and probe technique. ${ }^{10} \mathrm{~A}$ dye laser was pumped by a frequency doubled Nd:YAG running at $76 \mathrm{MHz}$. The dye laser output typically gave 6-7 ps pulses over a tuning range from around 800 to $960 \mathrm{~nm}$, using styryl $9 \mathrm{M}$ and styryl 13 dyes. The output of the dye laser is split into pump and probe beams with the probe beam traveling along a path which includes a variable delay line under computer control. This system allows the arrival of the probe pulse to be controlled from $-1 \mathrm{~ns}$ before, to $8 \mathrm{~ns}$ after the arrival of the pump pulse which excites the nonlinearity in the sample. The pump and probe are chopped at different frequencies and the nonlinear signal is detected with a photodiode and two lock-in amplifiers in series. The nonlinear signal is due to the change in probe transmission as a result of the change in absorption due to carriers injected by the pump pulse.

Figure 2 shows two pump-probe decays taken at average pump powers of $1 \mathrm{~mW}$, or less. The signal can be seen to rise from a thermal background signal at negative delays to a peak at zero delay, followed by a decay back toward the background signal. The background thermal signal has been measured as constant over the repetition rate of the laser. The rise in signal at zero delay is due to absorption bleaching in the quantum wells due to the production of carriers and the resultant nonlinear effects, ${ }^{11}$ such as fermion exchange, Coulomb screening, and phase space filling. By fitting the decay of this signal the carrier lifetime can be measured. Figure 2(a) shows the nonlinear decay for the
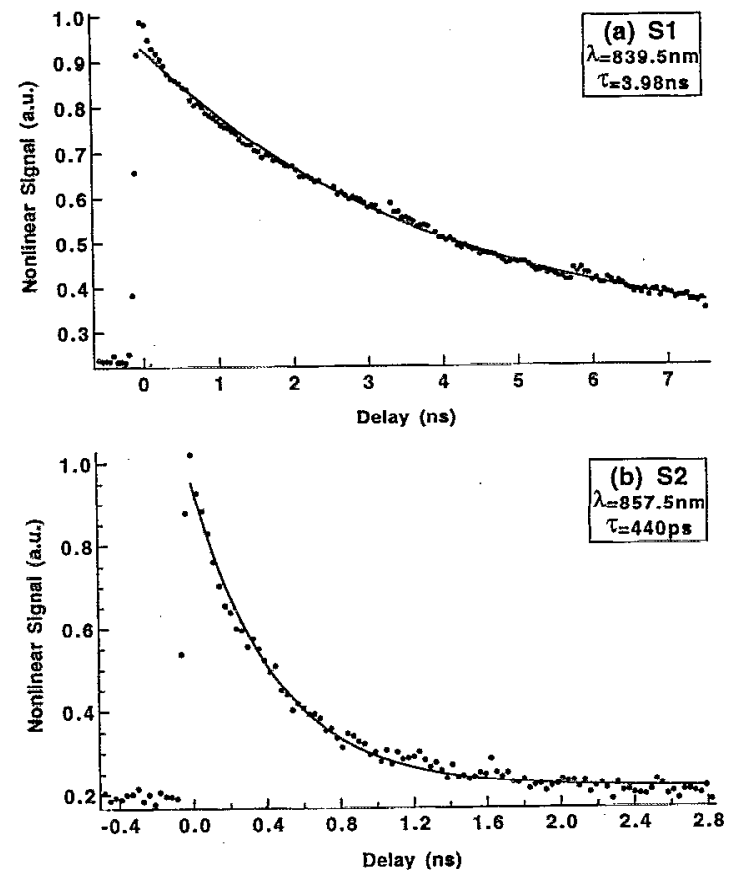

FIG. 2. Nonlinear pump-probe decays in $\mathrm{S} 1$ and $\mathrm{S} 2$. The signals can be seen to rise at zero delay, from the thermal background signal at negative delay, and subsequently decay back to the background level. Decays have been fitted to an exponential, shown with a solid line.

GaAs sample S1, which fits well to an exponential with a 3.98 ns time constant. Figure 2(b) shows a typical decay from one of the strained samples, in this case $\$ 2$, which fits an exponential of $440 \mathrm{ps}$. The lifetimes measured in all the samples discussed in this letter are shown in Table I.

Before considering the effects of strain on the samples we should consider the consequences of etching. Transmission measurements on samples $S 4 a$ and $S 4 b$, etched and unetched, respectively, show the exciton to be present at $927 \mathrm{~nm}$, indicating no difference in any strain relief that may be present in these samples. There is also no change in the lifetime measured in the etched and unetched samples with lifetimes of 553 and 552 ps, respectively. We can conclude that for the 8 period samples, etching does not adversely affect the strain or the lifetime measurements. None of the 40 period samples was etched.

The main focus of this letter is to investigate the effect of varying strain on the carrier lifetime in InGaAs quantum wells. Although the lifetime in S1 is short, as compared to typical GaAs samples, the lifetime decreases by an order of magnitude, approximately 4 to $0.5 \mathrm{~ns}$, as indium is introduced into the wells. Once the indium is present there is no further substantial change in the lifetime of the carriers with indium concentration, see Table $\mathrm{I}$. The lifetime in $\mathrm{S} 2$ is a little shorter than in $\mathrm{S} 3$ or $\mathrm{S} 4 \mathrm{a} / \mathrm{S} 4 \mathrm{~b}$, but as there is no definite trend with indium concentration we believe this difference in lifetime is not of fundamental importance. The increase in indium concentration, from $5 \%$ to $15 \%$, results in an increase in strain so we can conclude that the lifetime is, in general, unaffected by the alloy concentrations, or the amount of strain in the material.

As the number of periods is increased from 8 (S4) to 
40 (S5) the presence of strain relief is demonstrated by the shift of the exciton from 927 to $933 \mathrm{~nm}$, respectively, (see Fig. 1). The lifetimes in S4 and S5 are 552 and 564 ps, respectively. We can conclude that the strain relief present in these samples, and the changes in the material structure associated with strain relief such as dislocations, do not adversely affect the carrier lifetime. These results are somewhat surprising in that lifetime measurements, along with a decrease in the photoluminescence (PL) efficiency, ${ }^{8}$ have been suggested as a probe for detecting dislocations. Large scale production of dislocations in the process of catastrophic strain relief may indeed affect lifetime measurements, but the dominant carrier loss/recombination process in these samples is little affected by the strain relief.

The effect of harrier thickness is demonstrated by $\mathrm{S} 5$ and S6 with barrier thicknesses of 8 and $25 \mathrm{~nm}$, respectively. S6 was grown with thicker barriers, $25 \mathrm{~nm}$ as opposed to $8 \mathrm{~nm}$ in S1-S5. Thicker barrier structures are generally grown because of the decrease in low temperature (PL) linewidths that are reported for such samples. ${ }^{12}$ PL linewidths of 16 and $8 \mathrm{MeV}$ were measured at $10 \mathrm{~K}$ in S5 and S6, respectively, demonstrating the PL improvement which is often taken as an indication of material improvement. However, the thicker barrier structure does not affect the lifetime in S6, yielding a value of $570 \mathrm{ps}$. The lifetime was also measured in the AFPM, S7. This sample was grown by MBE and has the same indium concentration as $\mathrm{S} 3$ but with GaAs barriers and thicker wells. The mirror layers should not affect the measurements apart from the pump-probe experiment being carried out in reflection. The GaAs barriers result in much more shallow wells, making thermal loss from the well into the barriers a significant loss mechanism. For this reason material has been grown more recently with AlGaAs barriers to provide much deeper wells and minimize thermal loss. The lifetime measured in this sample was measured as 440 . ps. Both these results show that the use of deeper wells or thicker barriers does not significantly improve the carrier lifetime.

In conclusion, we have shown that the introduction of indium causes a dramatic decrease in the carrier lifetime by an order of magnitude. Contrary to what might be expected for nonlattice-matched material, once the indium concentration in the wells is $5 \%$ or more, the dominant carrier loss/recombination process in the InGaAs multiple-quantum-well materials is unaffected by the strain, strain relief, or barrier design. Possible recombination paths include defect centers, as a result of indium presence, or recombination centers at the well/barrier interfaces. Kirby et al. ${ }^{13}$ report that the well width dependence of the PL linewidth in strained materials, similar to those reported on in this letter, is inconsistent with monolayer fluctuations at the interfaces as seen in unstrained GaAs quantum wells. This may indicate that the interface formation process is different in strained pseudomorphic materials. It is possible that this interface formation process may also affect the lifetime. Further investigations are planned to discover the origin of carrier loss/ recombination in these samples, and whether the dramatic change in the lifetime occurs for indium concentration in the impurity or alloy range. These results are of great importance as they show that it is possible to design and grow strained layer devices that may require large number of wells, resulting in strain relief but without adversely affecting the carrier lifetime. The removal of any defects may in addition lead to match better device operating characteristics.

This work was carried out as part of FOCUS, ESPRIT Project No. 3180. The authors would like to thank Professor G. Parry and the technical staff of the Digital Optics Group at University College London for their assistance with the sample etching.

${ }^{1}$ B. Pezeshki, D. Thomas, and J. S. Harris, IEEE Photon. Technol. Lett. 2, 807 (1990).

${ }^{2}$ T. E. Sale, J. Woodhead, A. S. Pabla, R. Grey, P. A. Claxton, P. N. Robson, M. H. Moloney, and J. Hegarty, Appl. Phys. Lett. 59, 1670 (1991).

${ }^{3}$ M. H. Moloney, J. F. Heffernan, J. Hegarty, J. Woodhead, P. A. Claxton, and R. Grey, Conference on Lasers and Electro-Optics, Baltimore, MA, 1991, OSA Technical Digest Series (Optical Society of America, Washington, DC, 1991), Vol. 10, p. 46.

${ }^{4}$ P. L. Gourley, J. J. Wiczer, T. E. Zipperian, and L. R. Dawson, Appl. Phys. Lett. 49, 100 (1986).

${ }^{5}$ R. Jin, K. Okada, G. Khitrova, H. M. Gibbs, M. Pereira, S. W. Koch, and N. Peyghambarian, Appl. Phys. Lett. 61, 1745 (1992).

${ }^{6}$ E. Yablonovitch and E. O. Kane, J. Lightwave Tech. 6, 1292 (1988).

${ }^{7}$ R. L. Williams, M. Dion, F. Chatenoud, and K. Dzurko, Appl. Phys. Lett. 58, 1816 (1991).

${ }^{8}$ I. J. Fritz, S. T. Picraux, L. R. Dawson, T. J. Drummond, W. D. Laidig, and N. G. Anderson, Appl. Phys. Lett. 46, 967 (1985).

${ }^{9} \mathrm{H}$. Asai and K. Oe, J. Appl. Phys. 54, 2052 (1983).

${ }^{10}$ W. H. Knox, R. L. Fork, M. C. Downer, D. A. B. Miller, D. S. Chemla, C. V. Shank, A. C. Gossard, and W. Wiegmann, Phys. Rev. Lett. 54, 1306 (1985).

${ }^{11}$ S. Schmitt-Rink, D. S. Chemla, and D. A. B. Miller, Adv. Phys. 38, 89 (1989).

${ }^{12}$ R. Grey, J. P. R. David, P. A. Claxton, F. Gonzalez Sanz, and J. Woodhead, J. Appl. Phys. 66, 975 (1989).

${ }^{13}$ P. B. Kirby, J. A. Constable, and.R. S. Smith, Phys. Rev. B 40, 3013 (1989). 